\title{
Fan-shaped body neurons are involved in period-dependent regulation of long-term courtship memory in Drosophila
}

\author{
Takaomi Sakai, ${ }^{1,4}$ Show Inami, ${ }^{1}$ Shoma Sato, ${ }^{1}$ and Toshihiro Kitamoto ${ }^{2,3}$ \\ ${ }^{1}$ Department of Biological Sciences, Tokyo Metropolitan University, Tokyo 192-0397, Japan; ${ }^{2}$ Department of Anesthesia, \\ University of lowa, lowa City, lowa 52242, USA; ${ }^{3}$ Interdisciplinary Graduate Programs in Genetics and Neuroscience, \\ University of lowa, lowa City, lowa 52242, USA
}

\begin{abstract}
In addition to its established function in the regulation of circadian rhythms, the Drosophila gene period (per) also plays an important role in processing long-term memory (LTM). Here, we used courtship conditioning as a learning paradigm and revealed that (1) overexpression and knocking down of per in subsets of brain neurons enhance and suppress LTM, respectively, and (2) suppression of synaptic transmission during memory retrieval in the same neuronal subsets leads to defective LTM. Further analysis strongly suggests that the brain region critical for per-dependent LTM regulation is the fan-shaped body, which is involved in sleep-induced enhancement of courtship LTM.
\end{abstract}

[Supplemental material is available for this article.]

The Drosophila period (per) gene was identified as the first clock gene that plays a critical role in regulation of circadian rhythm (Konopka and Benzer 1971). Subsequent studies of per and other clock genes in Drosophila as well as in mammals led to the evolutionarily conserved molecular model of circadian timekeeping mechanisms, which revolutionized our understanding of the biological clock at the molecular level (King and Takahashi 2000; Hall 2003). We previously reported a surprising finding that, in addition to its well-known roles in circadian rhythm regulation, per is also involved in formation of long-term memory (LTM) induced by courtship conditioning (Sakai et al. 2004). Courtship conditioning is an established learning paradigm in Drosophila, in which male flies that have courted unreceptive, nonvirgin females subsequently suppress their courtship behavior even toward receptive virgin females (Siegel and Hall 1979; Mehren et al. 2004; Sakai et al. 2004; Griffith and Ejima 2009; Ishimoto et al. 2009). One-hour conditioning generates "courtship memory" detectable for several hours as an experience-dependent reduction in courtship activity (Siegel and Hall 1979), whereas 7-h conditioning results in LTM lasting at least $5 \mathrm{~d}$ (Sakai et al. 2004; Ishimoto et al. 2009). per-null mutant males show normal short-lasting courtship memory (Jackson et al. 1983; Gailey et al. 1991; Sakai et al. 2004). Interestingly, however, we found that they are defective in formation of courtship LTM (Sakai et al. 2004). In contrast to per, mutations in other clock genes do not affect courtship LTM (Sakai et al. 2004). Our results indicate that per plays a role in courtship LTM independently of its function as the core oscillator of circadian clock. The adverse effect of per mutations on courtship LTM was recently confirmed by Donlea et al. (2009). Furthermore, Chen et al. (2012) have shown that per, but not other clock genes, is required for LTM induced by Drosophila olfactory associative learning, suggesting the general role of per in control of different types of LTM.

\footnotetext{
${ }^{4}$ Corresponding author

E-mail sakai-takaomi@tmu.ac.jp

Article is online at http://www.learnmem.org/cgi/doi/10.1101/lm.028092.112.
}

To better understand the mechanisms underlying the novel function of per in memory processing, it is essential to identify the neuronal substrates that are responsible for per-dependent regulation of LTM. To address this issue, we used the enhancing effect of per overexpression on courtship LTM. Our previous study has demonstrated that 5 - $\mathrm{h}$ conditioning is insufficient for the formation of courtship LTM in wild-type males, but the same conditioning protocol becomes sufficient when per is temporarily but ubiquitously expressed using the $h s p 70$ promoter prior to 5 -h conditioning (Sakai et al. 2004). In the present study, wild-type per was overexpressed using the GAL4/UAS system to identify particular neuronal subsets important for per-dependent regulation of LTM (see Supplemental Material for detailed experimental protocols).

Particular attention was paid to the fan-shaped body (FB) of the central complex for the following reasons: First, per is likely expressed in FB neurons because the genomic per regulatory region drives reporter gene expression in this neuronal subset (Kaneko and Hall 2000). Second, the FB is known to process different types of memory. For example, visual pattern memory requires expression of functional rutabaga adenylyl cyclase-a proposed molecular integrator critical for memory formation-in the FB neurons (Liu et al. 2006; Pan et al. 2009). In addition, CaMKII activity in the FB neurons is necessary for courtship memory (Joiner and Griffith 1999). Third, Donlea et al. (2011) have reported that consolidation of courtship LTM is facilitated when the FB neurons are artificially activated immediately after conditioning using the temperature-gated cation channel TRPA1 (Donlea et al. 2011).

To examine whether per overexpression in the FB neurons leads to LTM enhancement, we used an enhancer trap line, OK348, which drives GAL4 expression mainly in a subset of the FB neurons (Fig. 3A, below; Connolly et al. 1996). Indeed, when per was overexpressed with OK348, 5-h conditioning became sufficient for the formation of courtship LTM (Fig. 1A, OK348/2-4), as was observed when per was ubiquitously expressed using the hsp70 promoter (Sakai et al. 2004). Similarly to wild-type males, control males showed courtship LTM after 7-h conditioning but not after 5-h conditioning (Fig. 1B, +/2-4 and OK348/+), 
A

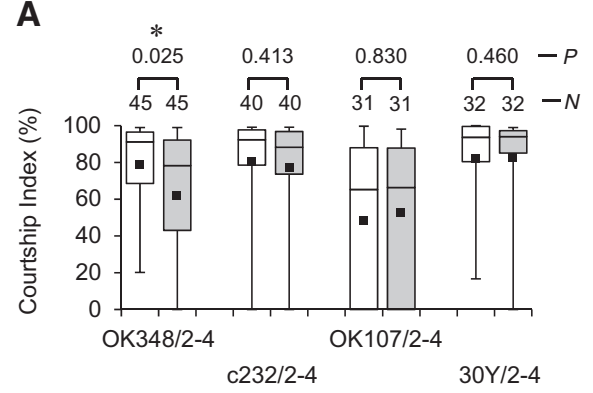

C

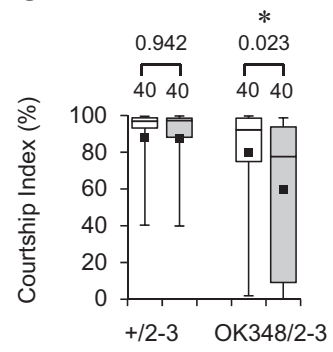

B

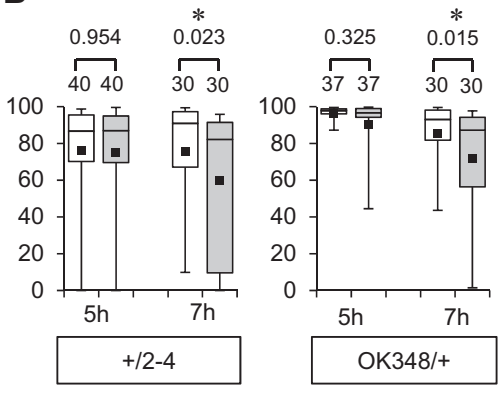

D $\quad$ E
E

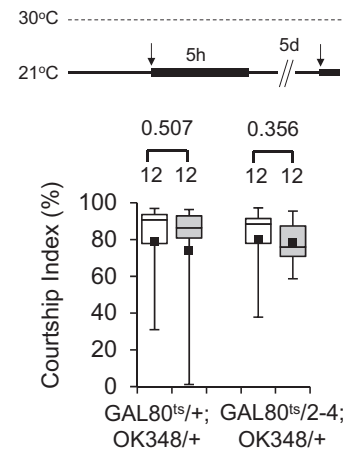

Figure 1. per overexpression in OK348-positive neurons enhances courtship LTM. In each box plot, the box encompasses the interquartile range. A line is drawn at median and vertical bars corresponding to the 10th and 90th percentiles. Each square within a box is the mean. White and gray boxes show the Cls of naive and conditioned males, respectively. In the labels for the $x$-axis, "2-4" and " $2-3$ " represent UAS-per2-4 and UAS-per2-3, respectively. (A) 5-d memory after 5-h conditioning in OK348/UAS-per2-4, c232/UAS-per2-4, OK107/UAS-per2-4, and 30Y/UAS-per2-4 males. ( $P$ ) Probability; $(N)$ sample size; $\left({ }^{*}\right)$ $P<0.05$. (B) 5-d memory after 5-h and 7-h conditioning in +/UAS-per2-4 and OK348/+ males; $\left({ }^{*}\right)$ $P<0.05$. (C) 5-d memory after 5-h conditioning in +/UAS-per2-3 and OK348/UAS-per2-3 males; (*) $P<0.05$. (D) 5-d memory in tub-GAL80 ${ }^{\text {ts }} /+$; OK348/+ and tub-GAL80 ${ }^{\text {ts }} /$ UAS-per2-4; OK348 $/+$ males. Males were kept at $21^{\circ} \mathrm{C}$. Four hours before conditioning, males were transferred to an environment of $30^{\circ} \mathrm{C}$ (restrictive temperature), and subsequently conditioned for $5 \mathrm{~h}$ at this temperature. Experimental paradigms are indicated above the graphs. The first and second arrows indicate the beginning of the 5-h conditioning and the 10-min test, respectively; ( $\left.{ }^{*}\right) P<0.01$. (E) 5-d memory after 5-h conditioning in tub-GAL80 ${ }^{\text {ts }} /+$; OK348/+ and tub-GAL80 ${ }^{\text {ts }} /$ UAS-per2-4; OK348/+ males. All experiments were carried out at the permissive temperature $\left(21^{\circ} \mathrm{C}\right)$.

indicating that the memory enhancement is due to overexpression of per in OK348-positive neurons. The LTM enhancement was also observed in combination of OK348 with a second UAS-per transformant (Fig. 1C, OK348/2-3).

The mushroom body $(\mathrm{MB})$ is a brain structure centrally important for olfactory memory (Davis 2005; Margulies et al. 2005) and courtship memory (Joiner and Griffith 1999; McBride et al. 1999; Ishimoto et al. 2009). However, the MB does not seem to be a critical brain region for per-dependent courtship LTM, because per is not likely expressed in neurons comprising the MB (Kaneko and Hall 2000). Consistently, LTM enhancement was not observed when per expression was directed to the MB using OK107 or 30Y (Fig. 1A, OK107/2-4 and 30Y/2-4). Likewise, overexpression of per in the ellipsoid body of the central complex using c232 did not lead to enhancement of courtship LTM (Fig. $1 \mathrm{~A}, \mathrm{c} 232 / 2-4)$.

Next, we used the TARGET system (McGuire et al. 2003) to determine whether transient expression of per in adult OK348positive neurons is sufficient for the enhancement of courtship LTM. As shown in Figure 1D, the memory enhancement was observed when per expression was temporarily induced in the OK348-positive neurons prior to and during 5-h conditioning (Fig. 1D, GAL80 ${ }^{\text {ts }} / 2-4 ; \mathrm{OK} 348 /+\left[30^{\circ} \mathrm{C}\right]$ ). In contrast, control males did not display LTM enhancement (Fig. 1D, GAL80 ${ }^{\mathrm{ts}} /+$; OK348 $/+\left[30^{\circ} \mathrm{C}\right]$; Fig. $\left.1 \mathrm{E}, 21^{\circ} \mathrm{C}\right)$. These results indicate that overexpressed per in the OK348-positive neurons facilitates physiological processes that are critical for formation or consolidation of courtship LTM.

We previously demonstrated that the activity of per-positive neurons is required for courtship LTM: disruption of synaptic transmission from per-GAL4positive cells specifically blocks retrieval of LTM (Sakai et al. 2004). We, therefore, examined whether courtship LTM is also affected by conditional blockage of synaptic transmission from OK348-positive neurons. The temperature-sensitive dynamin mutation shibire ${ }^{t s 1}\left(s h i^{t s 1}\right)$ (Kitamoto 2001) was used in combination with OK348 to disrupt synaptic transmission from the OK348-positive neurons in a temperature-dependent manner. First, all experimental procedures were carried out at a constant temperature of either $25^{\circ} \mathrm{C}$ (permissive) or $30^{\circ} \mathrm{C}$ (restrictive). We found that LTM is disrupted only when the restrictive temperature was used in the presence of both OK348 and UAS-shi ${ }^{\text {ts } 1}$ (Fig. 2A). GAL4 and UAS control males showed normal courtship LTM at both $25^{\circ} \mathrm{C}$ and $30^{\circ} \mathrm{C}$ (Fig. $2 \mathrm{~B}, \mathrm{C}$ ). To further examine the specific role of synaptic transmission from OK348positive neurons, courtship LTM was analyzed in OK348/UAS-shits 1 flies after temperature was shifted from permissive to restrictive during three distinct experimental phases- the period for courtship conditioning (memory formation), the test period (memory retrieval), and the period in between (memory storage). When temperature was increased to $30^{\circ} \mathrm{C}$ during the test period (Fig. 2D, 25-25-30), courtship LTM was not observed. However, as long as the temperature was permissive during the test period, normal courtship LTM was detected irrespective of the temperature during the conditioning period and the period in between (Fig. 2D, 30-25-25 and 25-30-25). These results indicate that synaptic transmission from the OK348-positive neurons is required during retrieval, rather than formation or storage, of courtship LTM.

Although OK348-driven GFP expression was primarily observed in the FB of the central complex (Fig. 3A, triangle) as previously reported (Connolly et al. 1996), additional expression was detected in clusters of the cells in the lateral region of the brain (Fig. 3A, arrows). GFP expression was also detected in a similar lateral region when the GFP reporter was driven by OK107 and 30Y (Fig. 3C,D, arrows). per is expressed in pacemaker neurons called lateral neurons (LNs), which include PDF neuropeptide-expressing neurons (Hall 2003). To examine whether the GAL4-positive neurons in the lateral region are circadian pacemaker neurons, we carried out a colocalization analysis using an anti-PDF antibody. As observed in pdf-GAL4 (Fig. 3E,G), PDF immunoreactivity was detected in some OK348- or 30Y-positive neurons in the lateral region (Fig. 3G). However, there was no coexpression of GAL4 and PDF in OK107-positive neurons (Fig. 3G). 

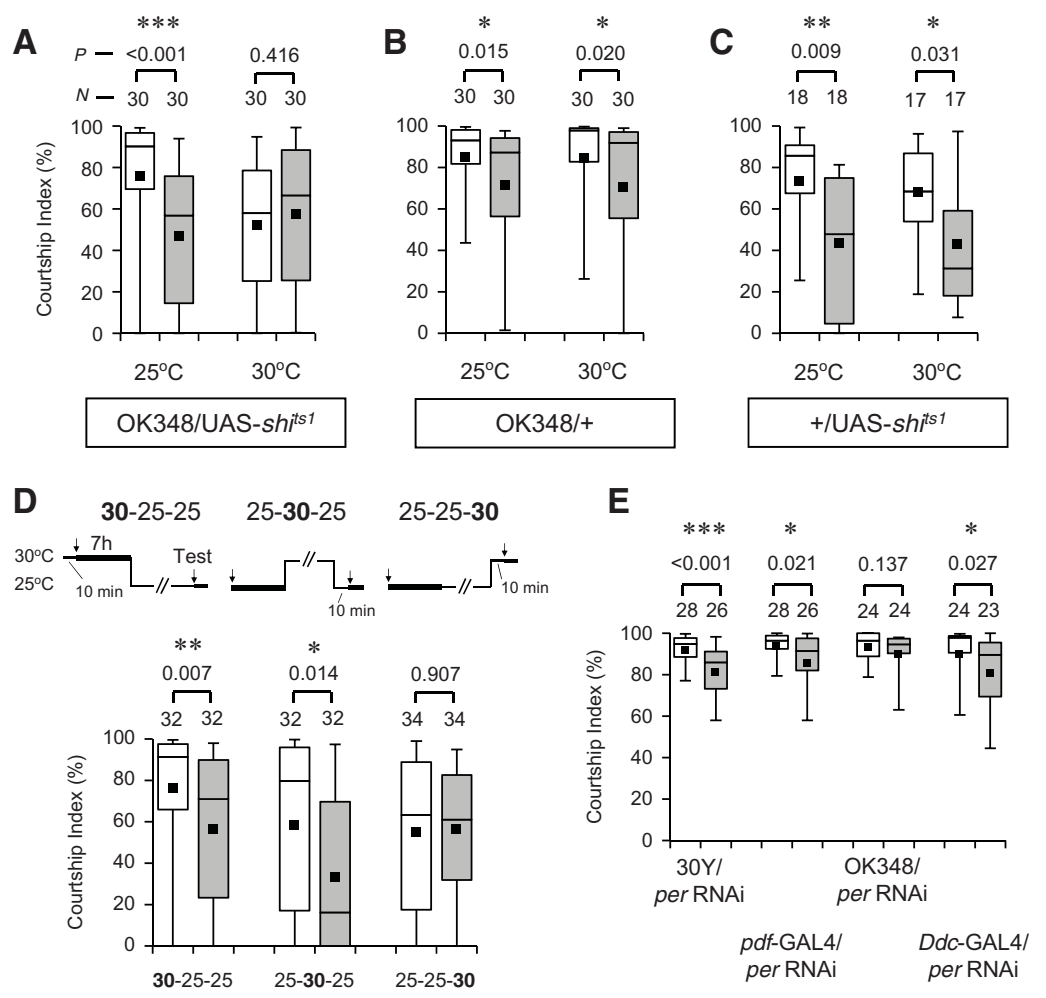

Figure 2. Effect of disruption of synaptic transmission in OK348-positive neurons on courtship LTM. (A-C) 5-d memory after 7-h conditioning. All procedures in the experiments were carried out at the permissive $\left(25^{\circ} \mathrm{C}\right)$ or restrictive $\left(30^{\circ} \mathrm{C}\right)$ temperature. OK348/UAS-shits $(A)$, OK348/ $+(B)$, and $+/$ UAS-shits ${ }^{t}(C)$ males were used. $(P)$ Probability; $(N)$ sample size; $\left({ }^{*}\right) P<0.05 ;\left(^{* *}\right) P<0.01 ;\left({ }^{* * *}\right)$ $P<0.001$. (D) 5-d memory after 7-h conditioning in OK348/UAS-shi ${ }^{\text {ts }}$ males. Experimental paradigms of temperature-shift are indicated above the graphs. The first and second arrows indicate the beginning of 7-h conditioning and 10-min test, respectively; $\left({ }^{*}\right) P<0.05 ;\left({ }^{* *}\right) P<0.01$. (E) 5-d memory after 7-h conditioning in $F_{1}$ males between UAS-per RNAi and 30Y, pdf-GAL4, OK348, or Ddc-GAL4; $\left(^{*}\right) P<0.05$; $(* *) P<0.001$. also requires per in the DAL neurons, we suppressed per expression in the DAL neurons and examined the effect of this suppression on courtship LTM. DdcGAL4, which directs GAL4 expression in the DAL neurons (Fig. 3F; Chen et al. 2012), was used in combination with UAS-per RNAi to suppress per expression in the DAL neurons. As shown in Figure 2E, Ddc-GAL4/UAS-per RNAi males were found to display normal courtship LTM after 7-h conditioning. This result indicates that, unlike olfactory LTM, courtship LTM does not require per expression in the DAL neurons.

Here, we have demonstrated that courtship LTM is suppressed when per expression in OK348-positive neurons is inhibited (Fig. 2E) or when synaptic transmission from OK348-positive neurons is blocked during the memory retrieval phase (Fig. 2D). These results suggest the possibility that courtship LTM is stored in these neurons and that their activation leads to manifestation of memory. Although per is a central component of the intricate gene regulatory network for circadian rhythm (Hall 2003), it could also control expression of the genes critical for formation or consolidation of LTM. Donlea et al. (2011) have recently shown that conditional activation of the FB neurons induces sleep and that this treatment also leads to enhancement of LTM - the effect we observed in this study when per was overexpressed in OK348-positive neurons
Because OK348-positive neurons include PDF neurons (Fig. 3G), it is possible that per expression in these PDF neurons, but not FB neurons, is critical for courtship LTM. To investigate this possibility, per expression was suppressed in PDF neurons using per-RNAi with pdf-GAL4, and its effect on courtship LTM was examined. We found that 30Y/UAS-per RNAi and $p d f$-GAL4/UAS-per RNAi males showed normal courtship LTM after the standard 7-h conditioning (Fig. 2E). In contrast, OK348/UAS-per RNAi males showed defective courtship LTM (Fig. 2E). These results strongly indicate that OK348-positive neurons other than PDF neurons are required for per-dependent courtship LTM. Thus, it is most likely that, among OK348-positive neurons, FB neurons are responsible for per-dependent regulation of courtship LTM.

Recently, Chen et al. (2012) have reported that per expression in two dorsal-anterior-lateral (DAL) neurons is required for LTM formation induced by olfactory conditioning. To determine whether formation of courtship LTM
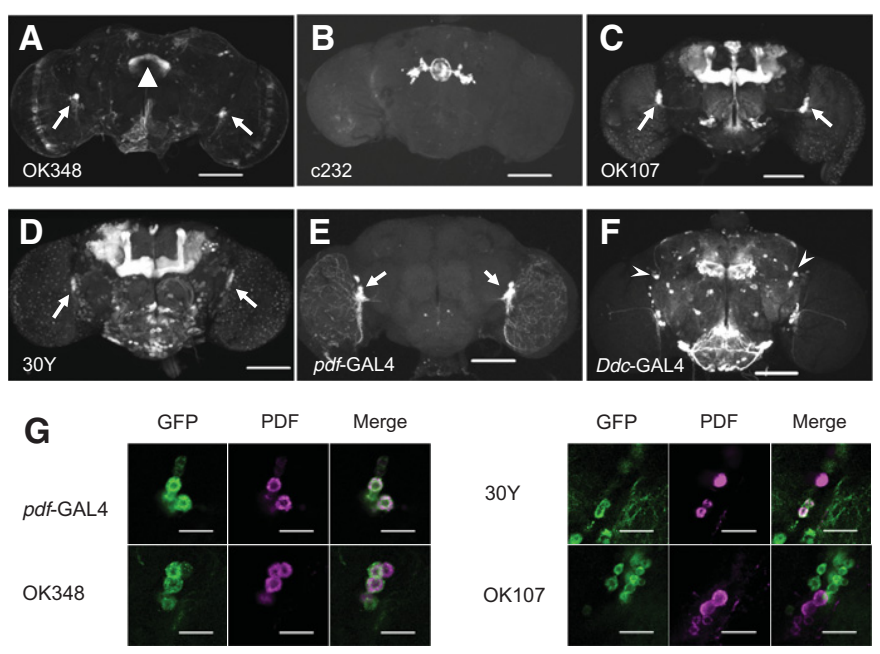

Figure 3. Confocal images of GAL4-positive neurons in the adult brain. $(A-F)$ Frontal views of the adult brain. $F_{1}$ males generated between UAS-mCD8::GFP and OK348 (A), c232 (B), OK107 (C), 30Y $(D)$, pdf-GAL4 (E), or Ddc-GAL4 $(F)$ were used. GFP fluorescence was observed under a confocal microscope (Carl Zeiss LSM710). Z sections were collected at 1- $\mu \mathrm{m}$ intervals and processed to construct projections through an extended depth of focus. Scale bars, $100 \mu \mathrm{m}$. (Triangle) Fan-shaped body $(A)$; (arrows) neurons in the lateral region $(A, C-E)$; (arrowheads) DAL neurons $(F)$. (G) GAL4-driven GFP in clusters of the cells in the lateral region of the brain (green) and PDF immunolabeling (magenta). $F_{1}$ males generated between UAS-mCD8::GFP and pdf-GAL4, OK348, 30Y, or OK107 were used. Scale bars, $20 \mu \mathrm{m}$. 
(Fig. 1). Thus, the intriguing possibility arises that per may mediate the enhancement of courtship LTM following FB activation and sleep induction. Future studies are expected to clarify the possible mechanistic link between nonclock functions of per in the FB neurons and regulation of sleep as well as LTM.

\section{Acknowledgments}

We thank Dr. Amita Sehgal for two UAS-per lines. UAS-per RNAi flies were provided by the NIG-FLY stock center. This study was supported by a Grant-in-Aid for Scientific Research on Innovative Areas "Systems Molecular Ethology" from the Ministry of Education, Culture, Sports, Science and Technology (to T.S.), the Sumitomo Foundation (to T.S.), and NIH grants (MH62684 and MH085081 to T.K.).

\section{References}

Chen CC, Wu JK, Lin HW, Pai TP, Fu TF, Wu CL, Tully T, Chiang AS. 2012. Visualizing long-term memory formation in two neurons of the Drosophila brain. Science 335: 678-685.

Connolly JB, Roberts IJ, Armstrong JD, Kaiser K, Forte M, Tully T, O'Kane CJ. 1996. Associative learning disrupted by impaired Gs signaling in Drosophila mushroom bodies. Science 274: 2104-2107.

Davis RL. 2005. Olfactory memory formation in Drosophila: From molecular to systems neuroscience. Annu Rev Neurosci 28: 275-302.

Donlea JM, Ramanan N, Shaw PJ. 2009. Use-dependent plasticity in clock neurons regulates sleep need in Drosophila. Science 324: 105-108.

Donlea JM, Thimgan MS, Suzuki Y, Gottschalk L, Shaw PJ. 2011. Inducing sleep by remote control facilitates memory consolidation in Drosophila. Science 332: 1571-1576.

Gailey DA, Villella A, Tully T. 1991. Reassessment of the effect of biological rhythm mutations on learning in Drosophila melanogaster. J Comp Physiol A 169: 685-697.

Griffith LC, Ejima A. 2009. Courtship learning in Drosophila melanogaster: Diverse plasticity of a reproductive behavior. Learn Mem 16: 743-750.

Hall JC. 2003. Genetics and molecular biology of rhythms in Drosophila and other insects. Adv Genet 48: 1-280.

Ishimoto H, Sakai T, Kitamoto T. 2009. Ecdysone signaling regulates courtship long-term memory formation in adult Drosophila melanogaster. Proc Natl Acad Sci 106: 6381-6386.
Jackson FR, Gailey DA, Siegel RW. 1983. Biological rhythm mutations affect an experience-dependent modification of male courtship behavior in Drosophila melanogaster. J Comp Physiol A 151: $545-552$.

Joiner MA, Griffith LC. 1999. Mapping of the anatomical circuit of CaM kinase-dependent courtship conditioning in Drosophila. Learn Mem 6: $177-192$.

Kaneko M, Hall JC. 2000. Neuroanatomy of cells expressing clock genes in Drosophila: Transgenic manipulation of the period and timeless genes to mark the perikarya of circadian pacemaker neurons and their projections. J Comp Neurol 422: 66-94.

King DP, Takahashi JS. 2000. Molecular genetics of circadian rhythms in mammals. Anпu Rev Neurosci 23: 713-742.

Kitamoto T. 2001. Conditional modification of behavior in Drosophila by targeted expression of a temperature-sensitive shibire allele in defined neurons. J Neurobiol 47: 81-92.

Konopka RJ, Benzer S. 1971. Clock mutants of Drosophila melanogaster. Proc Natl Acad Sci 68: 2112-2116.

Liu G, Seiler H, Wen A, Zars T, Ito K, Wolf R, Heisenberg M, Liu L. 2006. Distinct memory traces for two visual features in the Drosophila brain. Nature 439: 551-556.

Margulies C, Tully T, Dubnau J. 2005. Deconstructing memory in Drosophila. Curr Biol 15: R700-R713.

McBride SM, Giuliani G, Choi C, Krause P, Correale D, Watson K, Baker G, Siwicki KK. 1999. Mushroom body ablation impairs short-term memory and long-term memory of courtship conditioning in Drosophila melanogaster. Neuron 24: 967-977.

McGuire SE, Le PT, Osborn AJ, Matsumoto K, Davis RL. 2003. Spatiotemporal rescue of memory dysfunction in Drosophila. Science 302: $1765-1768$

Mehren JE, Ejima A, Griffith LC. 2004. Unconventional sex: Fresh approaches to courtship learning. Curr Opin Neurobiol 14: $745-750$.

Pan Y, Zhou Y, Guo C, Gong H, Gong Z, Liu L. 2009. Differential roles of the fan-shaped body and the ellipsoid body in Drosophila visual pattern memory. Learn Mem 16: 289-295.

Sakai T, Tamura T, Kitamoto T, Kidokoro Y. 2004. A clock gene, period, plays a key role in long-term memory formation in Drosophila. Proc Natl Acad Sci 101: 16058-16063.

Siegel RW, Hall JC. 1979. Conditioned responses in courtship behavior of normal and mutant Drosophila. Proc Natl Acad Sci 76: $3430-3434$.

Received August 9, 2012; accepted in revised form August 24, 2012. 


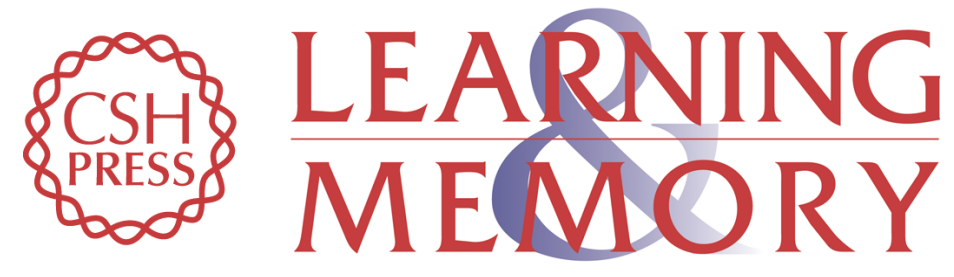

\section{Fan-shaped body neurons are involved in period-dependent regulation of long-term courtship memory in Drosophila}

Takaomi Sakai, Show Inami, Shoma Sato, et al.

Learn. Mem. 2012, 19:

Access the most recent version at doi:10.1101//m.028092.112

Supplemental
Material http://learnmem.cshlp.org/content/suppl/2012/11/07/19.12.571.DC1

References This article cites 23 articles, 12 of which can be accessed free at: http://learnmem.cshlp.org/content/19/12/571.full.html\#ref-list-1

License

Email Alerting Receive free email alerts when new articles cite this article - sign up in the box at the Service top right corner of the article or click here. 